\title{
EFFICIENT KEY PERFORMANCE INDICATOR CALCUlation
}

\author{
David Kuhlen ${ }^{1}$ and Andreas Speck ${ }^{2}$ \\ ${ }^{1}$ Datenlotsen Informations systeme GmbH, Technical Consultant, Beim Strohhause 27, \\ 20095 Hamburg, Germany \\ ${ }^{2}$ Christian Albrechts Universität zu Kiel, Head of the Business Information Technology \\ Group, Hermann-Rodewald-Straße 3, 24098 Kiel, Germany,
}

\begin{abstract}
In order to investigate the economic performance of business processes, simulations are performed. These simulations determine values of key performance indicators (KPIs ). We sought a valid solution to integrate KPIs in our Process Analysis Studio (PAS). Our investigation aims for a concept which describes a valid approach to deal with KPIs in multiple business process models.

In this paper, we present a valid concept of a KPI calculation model. We describe which types of KPIs are needed in order to give users a huge amount of freedom to investigate different aspects of the process performance. Using scenarios could empower comprehensive investigations of different interrelated aspects of the process. From the presented concept of scenarios special requirements are derived which have to be considered in a concept of a KPI calculation model.

The concept contributes to the discussion about the right design of a KPI calculation model. The presented approach could also be used in other process analysis solutions, in order to allow individual performance investigations.
\end{abstract}

\section{KEYWORDS}

process analysis, key performance indicators, system of scenarios

\section{Enhance Profitability}

In order to survive in a currently competitive environment, most companies must improve their business capabilities $(16,1773)$. In the past decade, multiple companies struggled, because their business environment has been changed beyond the limits of their capabilities $(7,11)$. Recovery of business success is only possible due to a comprehensive business process re-engineering initiative $(18,9)$. Processes have to be adapted permanently to prevent the company to drop behind its competitors $(9,75)$. Software developing companies (SDCs) need to enhance their abilities to control their process costs (10). However, controlling of financial performance information has become an increasingly complex task $(1,512)$. To measure the power of business success, key performance indicators (KPIs ) are used (20,3).

The whole efficiency of software developing companies is strongly linked with the efficiency of the core business process part: the production process (compare $(7$, p. 8$)$ ). Initiatives to improve 
International Journal of Software Engineering \& Applications (IJSEA), Vol.8, No.5, September 2017

the process are compounded, because a common set of tools and tactics is often unknown $(7,4)$. Management which is mainly interested in performance and reengineering, requires a tool that does not restrict their freedom to make rushed decisions $(1,514)$. As Cai et al. explained, it is important to describe the mutually dependent relationships among KPIs , and to optimize their accomplishment, based on their complex interdependence to assist the enhancement of process performance $(1,514)$. An analysis needs to be established to determine internal cost-KPIs and compare them with success- KPIs. Process improvement is a necessary step for most companies. The improvement consists of multiple decisions on the new design of the business process. Constructing a new process is equal to every investment decision - companies have to keep the balance between costs and success. Key performance indicators facilitate the assessment of process models. Different models differ in multiple indicators. Variation of the model leads to changes in the structure of KPIs.

\section{RESEARCH THESIS}

In order to evaluate the performance of business processes, a technique is needed to calculate KPIs during the process design. Such a technique needs to enhance existing methods of process simulation. A valid service architecture enables the calculation of KPIs on the fly during the process simulation. The purpose of this approach is the construction of a solution which facilitates the calculation of individual key performance indicators.

\section{KPI BASED PROCESS CONTROLLING}

Often a generic set of key performance indicators misses specific indicators that match to the selected process $(9,75)$. Key performance indicators could be derived from business process goals $(9,75)$. The derivation of KPIs is structured, compared to the re-engineering itself. Known from the design of IS, re-engineering can be difficult $(27,313)$.

Measurement and improvement are big challenges in the field of performance management (1, 513). To find critical KPIs of the improvement purpose, an optimization problem must be solved due to calculations $(1,514)$. Simulation would be used to measure values of key performance indicators like cycle time, output or efficiency $(20,3)$. The system of key performance indicators is a complex statistical model which has to be understood before the process might be changed ( 2 , 245). However, the relations within the statistical model are a subject of investigation. These dependencies are not understood, because the effects within the process model are wide and(sometimes) unpredictable.

Even if mathematical analysis models are important to measure the performance in experiments, they do not have received much attention in business process re-engineering and therefore, this area needs further development as Gunasekaran explained $(6,2533)$.

\section{RELATED WORK}

The concentration on processes could improve business success. The economic claims on successful processes are well elaborated. In 1993 Hammer and Champy wrote their publication about re-engineering the corporation (7). They concentrate their statements on the restructuring of business which also affects the redesign of process models. Their purposes have to be considered 
International Journal of Software Engineering \& Applications (IJSEA), Vol.8, No.5, September 2017

during modern designs of process models. In the same year, Davenport wrote his work on process innovation (2). This work also focuses on business-related demands in processes.

In order to derive an economic successful design of a process, its model has to be analysed. The analysis of process models by simulations was the topic of a multiple publication, e.g. by Hlupic et al., Silva and Chaix, Vergidis et.al, Lin et al. or Tan et. al. (8), (20), (25),(16), (22).

Economic analysis of processes needs to focus on key performance indicators. KPIs and their usage during process analysis was investigated by Van der Aalst and van Dongen ((24)), Field et al. ((5) or Spieß et al. ((21)) for example. Furthermore, Vom Brocke presented a publication on value-oriented process modelling which approaches the integration of financial aspects (26).

Some publications relate aspects of the design of simulation approaches. Prior researches of Tan et al. ((22) or Kuhlen and Speck ((13)) describe valid procedures to perform simulations. These approaches also focus on the construction of a software solution in general, in order to perform simulations.

\section{ANALYSIS}

\subsection{CONCEPTS OF KPI-BASED APPROACHES}

In practices, multiple companies engage in business process re-engineering initiatives. Reengineering is defined by Hammer and Champy to be a fundamental and radical redesign of business processes to achieve dramatic improvements contemporary measures of performance (7, 32). This definition emphasizes the mean performance measures have on radical business improvements.

Organizations seek to plan and control their work by the application of key performance indicators. Companies and their consultants endeavoured to establish a standard for process management maturity $(3,6)$.

With the help of a performance measurement standard, organizations can control their reengineering initiatives to determine if their investments in the process are valuable $(3,3)$.

In practice, the KPI system to calculate the return on investment is well known. This common ROI-system has been used in multiple different businesses. However, this system is often not adapted on the specific business case and not used in practices of business process modelling. It is meaningful to establish an own set of key performance indicators for each process type $(20,3)$.

A detailed evaluation is needed, to identify a valid set of key performance indicators for each core process $(20,3)$.

Regularly, the installation and utilisation of a set of key performance indicators is an exercise of the controlling. In this context, Speck et al. defined the tasks of monitoring and controlling to be the documentation, analysis and adjustment of business processes $(28,2)$.

The application of key performance indicators during the design of new business processes is hardly connected to the field of process simulation. In fact, simulations empower organizations to 
predict the impact, changes have on the overall performance. Process simulation revolutionises the way of thinking, merging business and IT $(20,9)$.

To express and differentiate impacts, performance indicators are needed. A calculation of such indicators during simulations needs to consider multiple aspects, like the prescriptions of the process model, the relationship between a network of KPIs and the experience of the organization (expressed in data of parameters). The simulation must consider the business progression of the organization to reproduce daily work of the company.

In practice, some barriers exist which hinder the application of a simulation. At first, the processes which are objects of interest in a re-engineering initiative could be complex and that's why managing (like simulating) of those is difficult $(7,16)$. Secondly, the relationship between performance indicators is also an object of consideration which could not be defined completely independent from process model. Multiple correlations of key performance indicators are unknown during the design of a process. For example, the correlation of requirements size (measured in function points) to the expected instance duration time is an important topic of own investigations. Such a performance strongly depends on the underlying model. As required by Vergidis, performance evaluations need to be integrated in the process design from the beginning $(25,12)$. Thirdly if data is incomplete, some relations and connections within the model could be lost $(4,3)$.

Key performance indicators have to be considered systematically during the analysis of a process. Their implementation in the process model gives the possibility to investigate a mathematical model of constraints. Having a connection between such a model and the process, enhances the possibilities to optimize both aspects: the system of performance indicators (and their relation) as far as the business constraints which are defined in the process model. To make the model applicable, it has to be implemented within a simulation approach. This makes it possible to measure the performance repeatedly to control whether the process is on track $(6,2539)$.

\subsection{Systematic Key Performance Indicators}

A set of key performance indicators could facilitate a systematic discussion as well as the analysis of organization processes. Most notably, business values are not considered structurally $(26,2)$. Business process models could be divided in multiple levels to describe the progression of value creation through the interaction of people and artefacts $(17,1)$. This model of interactions in combination with the state of the environment prescribes the performance of key business values. During the business process re-engineering, we seek to optimize the process, for example, by reducing its costs or optimizing another KPI $(2,6)$. The optimization of key performance indicators becomes the target of the BPR initiative. By having a system based on key performance indicators, it might be possible to derive sensible changes easier, to realize the required success.

A systematic approach to analyse organisations is process simulation. A simulation consists of model construction and execution, the analysis of performance and the evaluation of costeffective alternative scenarios $(20,6)$.

The application of structured performance measurements facilitates an automated business process optimization $(25,8)$. A system of key performance indicators which are interconnected 
International Journal of Software Engineering \& Applications (IJSEA), Vol.8, No.5, September 2017

with the process model could become the key of success in business process re-engineering. It gives the re-engineering a clear, reachable target and it helps to understand the way, how to reach the performance target. Prerequisite is aggregated economic process data (available in key performance indicators) to judge the profitability of process design $(26,9)$. Organizations need to assess their current situation before efficient changes are possible $(20,1)$.

An established system of key performance indicators simplifies the job of management. Management has to identify critical KPIs which need to be improved together with target values $(1,512)$.

Making use of a system of key performance indicators connected with the process model is a promising attempt. It promises to allow advanced scenario analyses $(20,7)$. Using the system of scenarios allows to test the impact of multiple different strategies on the profitability. It enhances the formulation of an ideal strategy which allows the organization to achieve an optimal constellation of values in the key performance indicators.

\section{Design of a KPI service architecture to enhance the Process ANALYSIS STUDio (PAS)}

\subsection{SYSTEM OF SCENARIOS}

Scenarios express possible constellations of situations which might happen in the future. They could be expressed as a set of key performance indicators.

Scenarios might help to find optimal constellations of parameters. The design of parameters has to facilitate the analysis, to find the fittest scenarios by comparing them. Scenarios help to test the impact of changes in the environment (the processes) on the behaviour of a system (also an organization).

To aid business process re-engineering initiatives, a systematic approach is needed. This systematic approach allows deriving new scenarios automatically.

To systematize scenarios, attributes need to be distinguished in such attributes which describe the scenario itself (parameters) and such attributes which contain the output of the scenarios after its execution (key performance indicators). The general approach consists of changing parameters in the model, execute the simulation and analyse the achieved result $(20,8)$. By the repetition of such procedures of manipulation, simulation and value analysis (MSA-Investigation), multiple scenarios could be tested and compared. The use of a simulation facilitates the decision-making process to predict the impact of further changes $(20,4)$. A common use case of such an investigation could be the comparison of costs and risks in different strategies, in order to aid management $(1,520)$.

The adaption of the scenarios-concept within the process analysis introduces an iterative analysis $((2,146))$ of multiple different scenarios.Multiple experiments would be executed to find the best constellation of parameters $(20,7)$.

Using scenarios could facilitate the business process re-engineering initiative, so that weaknesses within the process can be detected. To use scenarios within the analysis, the concept needs to be 
International Journal of Software Engineering \& Applications (IJSEA), Vol.8, No.5, September 2017

applied on the process model. Therefore, an object is necessary, to contain scenarios within the analysis. This object needs operations to derive scenarios, check whether scenarios already exist (equals) and to prove the scenario (execute).

\subsection{ReQUirements ON A GenERAl APPROACH}

To facilitate process analysis, an approach is necessary to consider scenarios in the simulation. The application of simulation is sensible to reduce risks within the business process reengineering $(8,1363)$. The adaption of simulation techniques needs to express the impact, process changes might have on systems (organizations) behaviour. A new approach which allows the calculation of scenarios in the process simulation, ought to fulfil multiple requirements. A good, structured method might be useful to analyse business processes $(19,32)$. Important requirements on a solution to facilitate process analysis need to be derived from the procedure of process analysis itself.

Lin et al. explained, that only if people have a good understanding about the structure and performance of a process, they know which factors account for which results $(16,1775)$. This limits the analysis on those people who have necessary knowledge on the process. The process often predicts possible achievable results. This results could be determined without much knowledge on the process. It would be possible to simplify the information about in- and output on simple process variables which could be used by everyone in the management.

Requirements on an approach are related to the need for input data, the integration of the approach into the simulation and on its obtained results. Baseline data is needed to express the current situation within the analysis $(2,138)$. Often, a system of key performance indicators (multiple indicators decomposed in metrics) is strongly connected to its process (to measure) and so, slight modifications of the process require changes in the KPI -model $(9,81)$. To facilitate changes of the underlying process model (within process analysis exercise) this issue should be addressed with a new approach.

A new approach needs to allow the measurement of the result for specific KPIs . Business process improvement should achieve enterprise-wide goals which often means that after an improvement, target values match the expectations $(9,75)$.

During different simulations, the parameters which should be changed, must be specified. Therefore, the approach needs to distinguish ceteris paribus parameters.

Furthermore, it is necessary to determine a calculation procedure to assess key performance indicators. This calculation method must be able to use the values of other key performance indicators. This leads to a higher level system of indicators. However, it also requires an environment which has to deal with situations if new process variables are introduced and used without a prior initialization. To simplify the approach, parameters should be initialized if they are used for the first time.

The introduction of a lazy-initialization (initialization only if the value is needed) has advantages and disadvantages. One of the disadvantages regards to the stability of calculated values. Process variables need to be calculated iteratively. A repetition of calculation has to lead to (situation-) adapted results. On the other side, process variables need to be stable within a simulation cycle. 
International Journal of Software Engineering \& Applications (IJSEA), Vol.8, No.5, September 2017

The right degree of stability differs between process variables. It depends on the variable and the aim of investigation. The approach needs a possibility to define the right stability for key performance indicators.

Furthermore, the approach should allow a fast calculation of key performance indicators. It might be positive if process variables which are unnecessary are not calculated every time (lazy initialization). On the other hand, this approach requires a fast calculation at access time.

Process performance measurement is a necessity for modern process-oriented organizations $(9$, 82).

To fulfil the above-mentioned requirements, it is necessary to start designing abstract classes. Therefore, a formal definition of the above mentioned Process Variable, Key Performance Indicator and Parameter are needed. These abstract classes can be used in the underlying application model. They should be integrated in the construction of reports, e.g. The last step consists of the implementation of concrete variables.

Normally, process performance measurement itself does not show which action is needed to improve the process $(9,82)$. However, the construction of scenarios helps to find optimal constellations of parameters. This may simplify the achievement of the target (optimal-) parameters. Therefore, the new approach needs to aid the formulation of a process vision, as Davenport explained, to set new targets $(2,127)$.

\subsection{CONCEPT OF A SOUND STRUCTURE}

In order to investigate the success of a business model, properties of its process have to be analysed $(23,8)$. As explained by Hammer and Champy, companies which aim for radical improvements, scrutinise the whole structure of their business model $(7,4)$. What business needs to undertake hereof, might be individual. To enhance process analysis, it is therefore necessary to analyse individual key performance indicators.

The definition of a process needs to be connected directly to the definition of key performance indicators. The KPIs must be assessed while the business model becomes parsed (11). To analyse the impact of changes (as a result of business investigations), simulations need to be repeated frequently (13). In the analysis, the division of KPIs should match the economic need (11).

KPIs enhance comparing multiple process versions, in order to judge on their profitability (12). Management would be guided due to the link of process and strategy by key performance indicators (14).

To realize a solution, we designed a model of operation to deal with KPIs . This design fits to the model of PAS . However, the business model of PAS also needs to consider some conventions.

Key performance indicators have no relation to concrete or singular objects. However, they could be assigned to classes of objects. We need to introduce a convention so that each $1: \mathrm{N}$ relation is solely being located at the targeting object. Therefore, we do not store lists at entities.

During a simulation, instances are processed through activities (vertexes) in PAS (14). To simplify the access on KPIs and their values, all objects which might carry KPIs need to extend 
the PAS Object class. For each PAS Object (based on its type) special KPIs could be defined. An overview of the KPI object model is given in the figure 1.

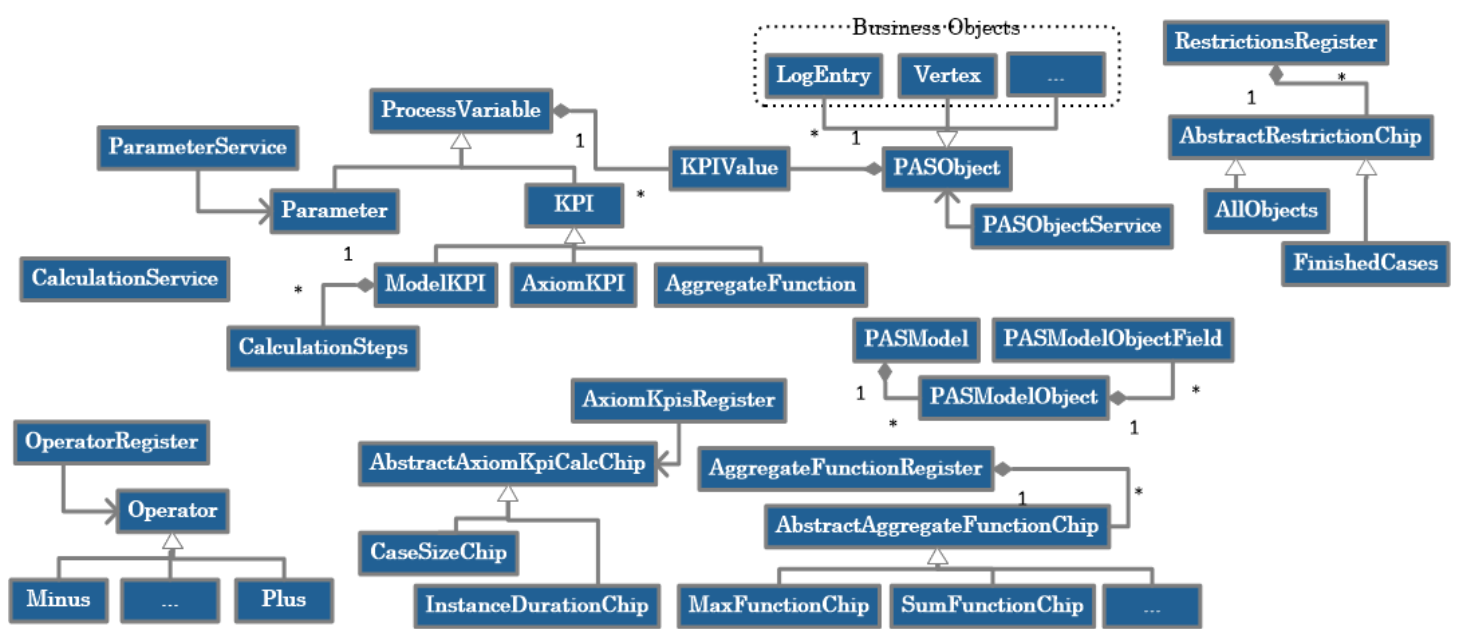

Figure 1: High level overview of the class model which belongs to the calculation of kpis

KPIs of the process model itself, might be related to actors (salary, worked hours, hours of absence) or to vertexes (worked hours, variable costs, number of operations...) or any other object which defines the operational sequence. The simulation bases on actors who act and record what they were carrying out (15). The actors operate in processes which consist of a list of flows (tuple of activities) (13). PAS simulation framework bases on a container which stores all relevant elements of the model to enhance reports and investigations (13). Different from our previous version of PAS (see (13)), instead a project represents the container, together with its PAS Objects and its Process Variables (see figure1).

As we are simulating the progression in different models (15), KPIs need to be related to different types of entities. KPIs which are related to progression elements, need to be predefined. The predefinition must be part of the application model of PAS. Progression elements emerge during the simulation. They are not a part of the process model, however, they play an important role in the simulation. KPIs might influence the behaviour of processes. In order to connect special KPIs with the business logic, pre-defined KPIs are important. However, some KPIs should be defined by the user. Furthermore, static KPIs are needed. It must be possible to value these KPIs by initiating the business process model.

Individual investigations need to interrelate selected attributes, to analyse the performance (13). During the simulation, a special service implementation needs to enable the calculation of KPIs . The calculation method creates a KPI Value for a PAS Object and a Simulation Cycle (see figure 1). The whole simulation consists of multiple cycles (13). First, we aim to repeat the calculation of KPIs in every simulation cycle. The calculation requires of an iteration through the objects and an iteration through the KPIs . As figure 2 shows, for each tuple of KPI and object the calculation has to be executed. The calculation differs, regarding to the type of the KPI . The procedure to perform the calculation has to be part of the business logic of PAS. 


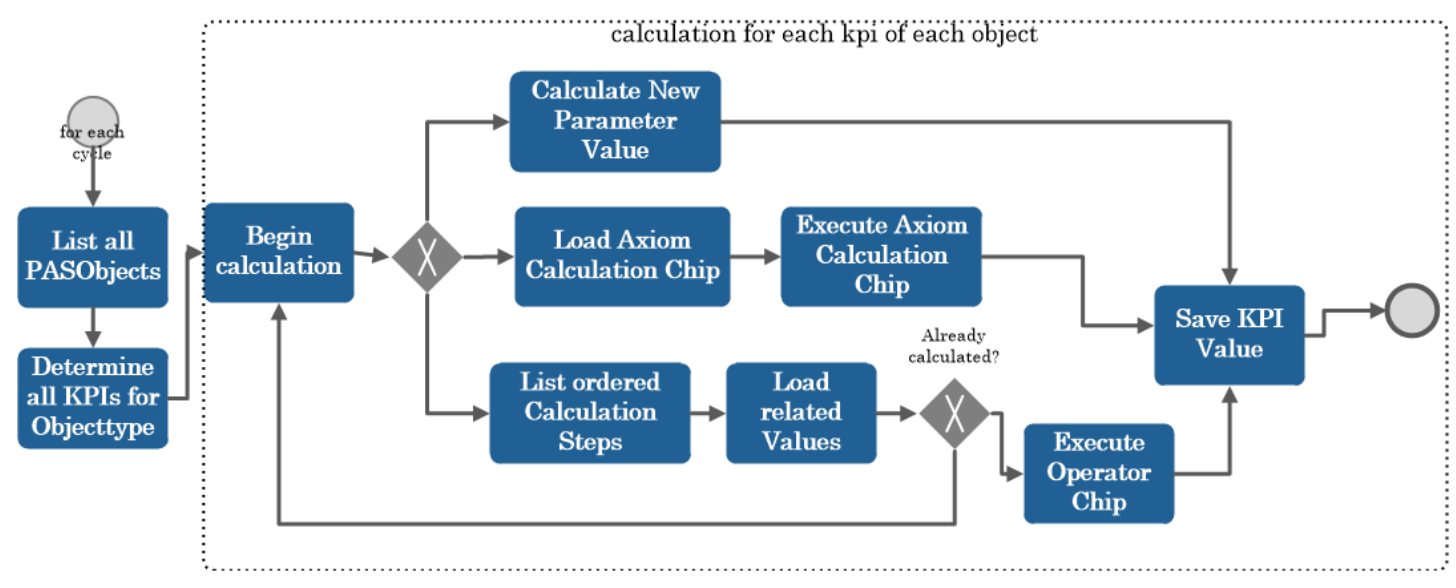

Figure 2: Procedure of the calculation of key performance indicators in processes

KPIs which belong to the user-defined model have a generic calculation procedure. Predefined KPIs (Axiom KPIs) ought to have an individual (- predefined -) calculation procedure. However, to standardize the handling of different types of KPIs, their definition has to be distinguished from their application (calculation). Therefore, pre-defined KPIs also need a definition which could be used to assign them to other KPIs calculation procedures. In order to calculate these predefined KPIs, their calculation procedure needs to be connected to their definition. This relation of axiom KPIs to the calculation logic would be established by system identifiers. To handle the predefined KPIs, a register is needed which stores all of them. The register of Axiom KPIs needs to create a definition for each predefined KPI. At the beginning of the application, PAS needs to make sure that for each Axiom KPI a definition will be created. This enables a consistent way to calculate KPIs.

The calculation of a KPI creates a KPI Value (see figure 2). This KPI Value is directly associated to a PAS Object (see figure 1). Results should be stored for each simulation cycle. If during the calculation a result of a KPI misses, (e.g. in case of combined KPIs), the missing KPI needs to be calculated first. Attention has to be given to cyclic dependencies.

In order to investigate business processes, PAS allows relating activities, actors and actions to achieve results (like instances and other progression elements) (13).

This leads to a model of business objects which are strongly connected to each other. The calculation of KPIs has to consider these $1: \mathrm{N}$ relations. The calculation of KPIs of one object, has to be able to access the KPIs of its related objects. In order to access KPIs of related objects, they need to be aggregated for all associated objects. As figure 1 shows, our calculation model provides Aggregate Functions. Each Aggregate Function serves as a KPI which also has a definition that is stored.

In order to access related KPIs, we need to use aggregate function (e.g. like sum(...), minimum(..), maximum(...), average(...), number(..)). These functions calculate a new KPI Value for a list of KPI Values.

The main challenge, regarding to Aggregate Functions, is to list the connected objects which belong to a superior object. The way how the objects are connected (especially the involved 
International Journal of Software Engineering \& Applications (IJSEA), Vol.8, No.5, September 2017

columns) is discretionary. This way needs to be stored in a relation. The algorithm in listing 1 returns a list of Model Objects. The object model, returned from listing 1, contains a list of associated types. For each type, multiple concrete objects are connected.

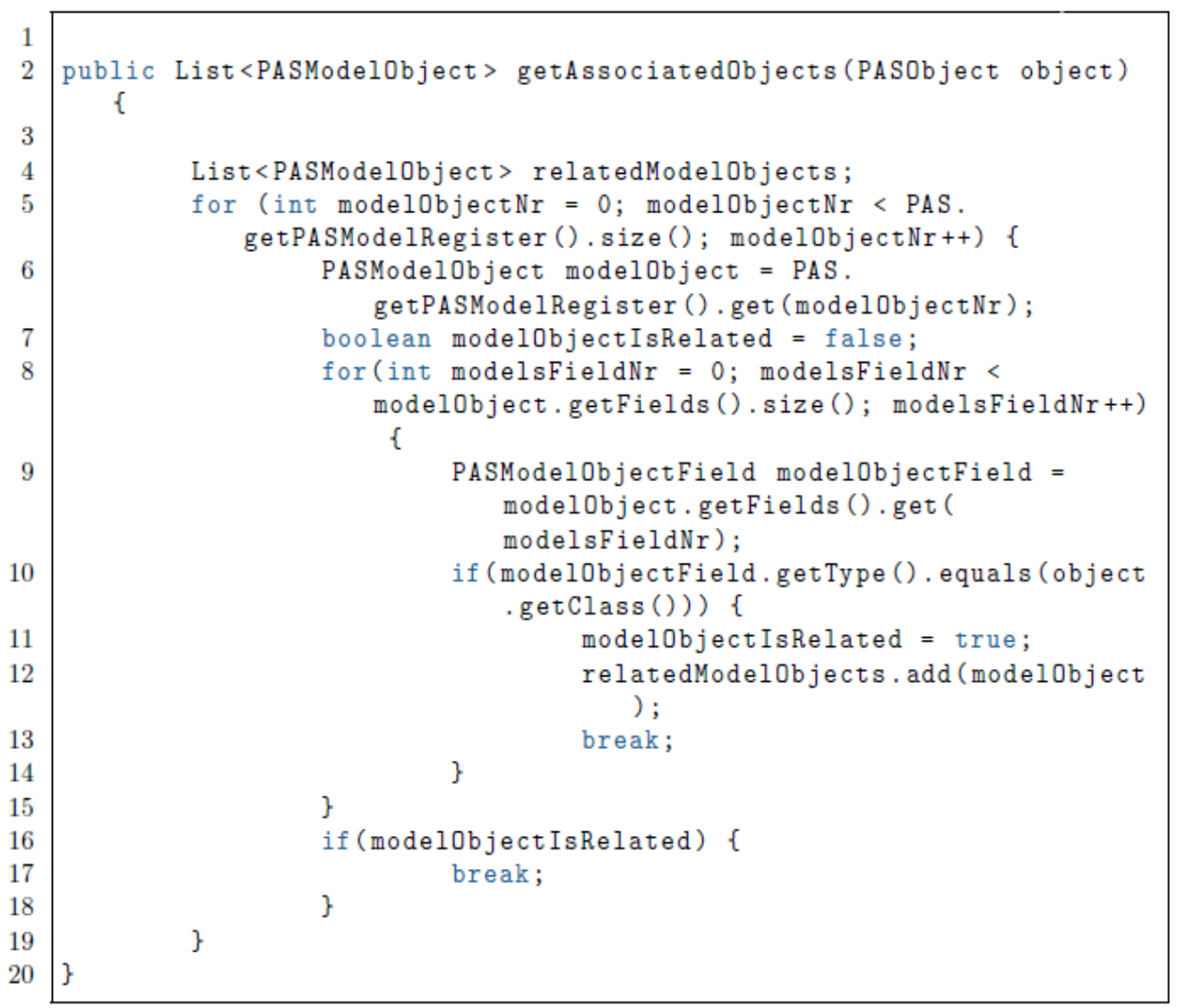

Listing 1: Pseudo code of an algorithm to collect all object Models which belong to a superior PASObject

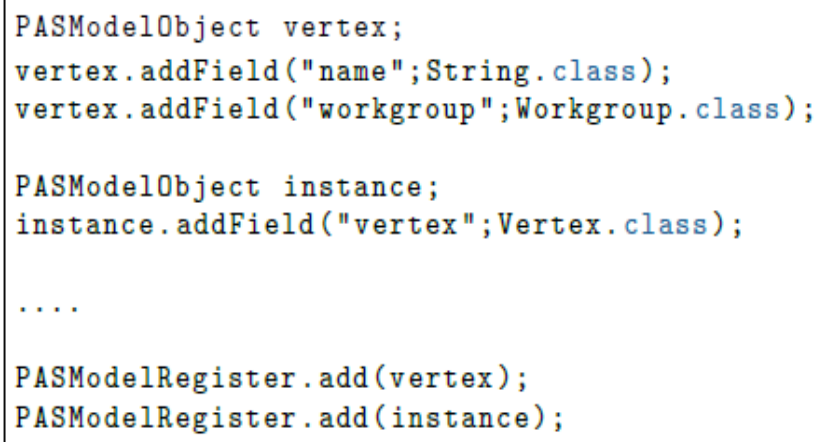

Listing 2: Pseudo code to illustrate the way of initiating the PASModel.

Each aggregate function has a calculation chip. This chip needs to operate on a set of PASObjects which are created by an AbstractRestrictionChip. 
In order to empower the user to isolate his investigations on a special group of PASObjects, we used restriction chips. We decided that predefined restriction chips as part of the business logic would be valuable in order to aid the user and to retain him from the complexity of the underlying model.

In further developments, a condition would be valuable to guide the execution of calculation steps. Such a condition could also be implemented on the basis of the object model. However, its usage has to be easy for the user. Therefore, its implementation needs to provide a list of possible values for each field. This should also consider different values from enumerations.

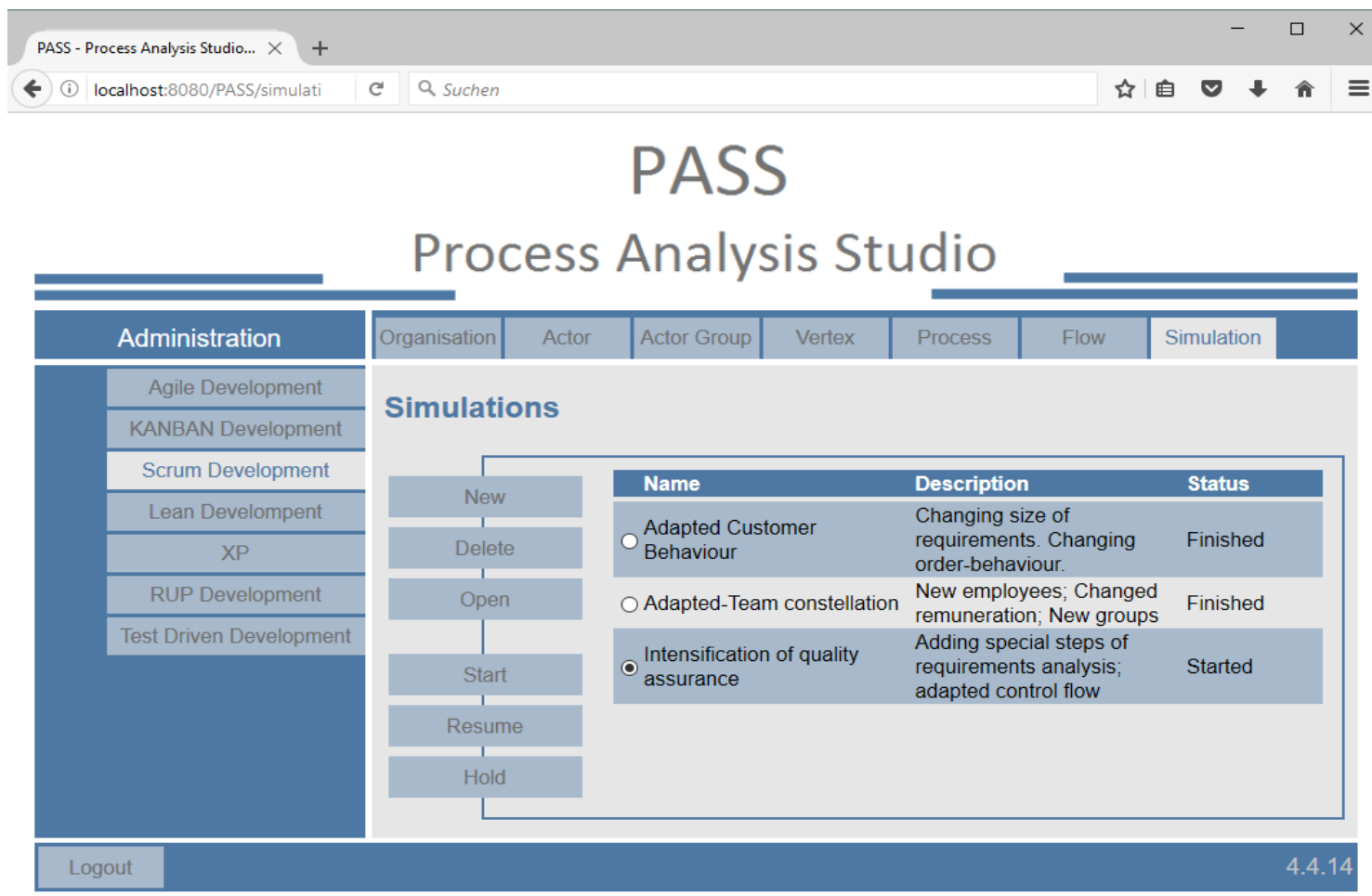

Figure 3: Screenshot of simulations in our software PAS

Figure 3 illustrates the interface of PAS . It shows a list of simulations which could be executed within a project in order to analyse the progression of its processes.

\section{CONCLUSION}

Business process analysis needs to include the calculation of key performance indicators. KPIs need to stay in close relation to the process model. Some KPIs have to be predefined. However, the user needs to control his investigations by including own KPIs. Because of the fact that objects are strongly interrelated in the process model, aggregate functions are necessary to facilitate the access on KPIs over relations. Furthermore, static KPIs are needed, to assess values directly while the model is created. In this way, four types of ProcessVariables are needed. 
International Journal of Software Engineering \& Applications (IJSEA), Vol.8, No.5, September 2017

Our investigations revealed a design of a KPI calculation model. The model allows the computation of multiple KPIs in different investigations. The design fits into a modern process analysis studio like PAS. It considers the division of application layers. If other process simulation applications seeked to implement a KPI calculation model, they could align on the presented approach. As a consequence, this paper might contribute on the discussion about how to implement a KPI-calculation approach.

The presented approach fits into all conceivable kinds of process models. However, it does not allow the calculation of all kinds of KPIs yet. In some cases, it might be imaginable that KPIs have to be calculated on the basis of conditions. The introduction of conditions is complex, for both: in the development and in the application of the solution. Certainly, the advanced calculations are imaginable (also with conditions) by editing the obtained KPIs from PAS with other tools (like Microsoft Excel). The presented approach copes the research thesis, presented in section. It demonstrates how to implement a sound solution which fulfils the thesis.

However, it could be of interest, to develop a solution to enable the calculation of KPIs based on conditions. Such an approach might base on a domain specific language, in order to enhance the possibilities for the user.

\section{REFERENCES:}

[1] Jian Cai, Xiangdong Liu, Zhihui Xiao, and Jin Liu. Improving supply chain performance management: A systematic approach to analyzing iterative kpi accomplishment.Decision Support Systems, 46(2):512-521, 2009. URL http://litis.univ-lehavre.fr/ serin/facade/uploads/Master/MIS6SupplyChain.pdf.

[2] Thomas $\mathrm{H}$ Davenport. Process Innovation - Reengineering Work through Information Technology.Havard Business School Press, Boston, Massachusetts, 1993. 27-36 pp. Ernst \& Young Center for Information Technology and Strategy.

[3] Thomas H Davenport. The coming commoditization of processes. Harvard business review, 83(6):100-108,2005.URL

http://www.researchgate.net/profile/Thomas_Davenport2/publication/7799378_The_coming_commo ditization_of_processes/links/ 53db93aa0cf2a76fb667a58b.pdf.

[4] María Fernández-Ropero, Ricardo Pérez-Castillo, and Mario Piattini. Challenges of business process model improvement after reverse engineering. 1st International Workshop on Software Evolution and Modernization(SEM2013), January $2013 . \quad$ URL http://alarcos.esi.uclm.es/per/mfernandez/papers/SEM13.pdf.

[5] Frank Field, Randolph Kirchain, and Richard Roth. Process cost modeling: strategic engineering and economic evaluation of materials technologies. Jom, 59(10):21-32, 2007. URL http://msl.mit.edu/publications/field_kirchaincm_stratevalmatls.pdf.

[6] A Gunasekaran and B Kobu. Modelling and analysis of business process reengineering.International Journal of Production Research, 40(11):2521-2546, 2002. URL http://www.umassd.edu/media/umassdartmouth/businessinnovationresearchcenter/publications/model ling_bpr.pdf.

[7] Michael Hammer and James Champy. Reengineering the Corporation A Manifesto for Business Revolution. HarperBusiness A Division of HarperCollinsPublishers, New York, NY, USA, 1993. 
International Journal of Software Engineering \& Applications (IJSEA), Vol.8, No.5, September 2017

URL

https://moodle-

res.unitec.ac.nz/pluginfile.php/192283/mod_resource/content/0/Reengineering_The_Corporation.pdf.

[8] Vlatka Hlupic and Stewart Robinson. Business process modelling and analysis using discrete-event simulation. In Proceedings of the 30th conference on Winter simulation, pages 1363-1370. IEEE $\begin{array}{llll}\text { Computer } & \text { Society } & \text { Press, } & 1998 .\end{array}$ http://citeseerx.ist.psu.edu/viewdoc/download?doi=10.1.1.1.1814\&rep=rep1\&type=pdf.

[9] Peter Kueng. Process performance measurement system: a tool to support process-based organizations. Total Quality Management, 11(1):67-85, 2000. URL http://www.researchgate.net/profile/Peter_Glassman2/publication/8180753_Measuring_the_quality_o f_physician_practice_by_using_clinical_vignettes_a_prospective_validation_study/links/0f31753bac 39895356000000.pdf.

[10] David Kuhlen and Andreas Speck. Wertanalyseverfahren für kundenanforderungen.In Erhard Plödereder, Lars Grunske, Eric Schneider, and Dominik Ull, editors,INFORMATIK 2014 Big Data Komplexität meistern, volume P-232 of Lecture Notes in Informatics (LNI) - Proceedings, pages 2317 - 2322. Gesellschaft für Informatike.V. (GI), Stuttgart, September 2014. ISBN 978-3-88579626-8. URL http://cs.emis.de/LNI/Proceedings/Proceedings232/2317.pdf. Thanks to Prof. Dr. Andreas Speck and Prof. Dr. Hinrich Schröder.

[11] David Kuhlen and Andreas Speck. Business process analysis by model checking. In 5th International Symposium on Data-Driven Process Discovery and Analysis SIMPDA 2015, pages 154-170. Ceravolo, Paolo and Rinderle-Ma, Stefanie, Vienna, Austria, December 2015. URL http://ceurws.org/Vol-1527/paper15.pdf.

[12] David Kuhlen and Andreas Speck. Project-economy. Dresden, Germany, 2016. Fachtagung Verwaltungsinformatik und Fachtagung Rechtsinformatik 2016, Gesellschaft für Informatik e.V. (GI), Lecture Notes in Informatics (LNI), Conference date September 21-23 2016, in print.

[13] David Kuhlen and Andreas Speck. The way of designing a simulation software in order to evaluate the economic performance in software development. In The 8th International Conference on Computer Modeling and Simulation, International Conference Proceedings Series by ACM, 2016. Conference date January 20-23 2017, in print.

[14] David Kuhlen and Andreas Speck. Magnitude economic effects of requirements in the development process. In SAI Computing Conference (SAI), 2017, Conference Proceedings Computing Conference 2017. IEEE, London, United Kingdom, 18-20 July 2017 2017. conference date 18-20 July 2017, in print.

[15] David Kuhlen and Andreas Speck. Economic output under the conditions of social freedom in software development. International Journal of Software Engineering \& Applications (IJSEA), (No. 6), November 2016. URL http://aircconline.com/ijsea/V7N6/7616ijsea02.pdf.

[16] Huiping Lin, Yushun Fan, and Stephen T Newman. Manufacturing process analysis with support of workflow modelling and simulation. International Journal of Production Research, 47(7):1773-1790, 2009.URLhttp:/www.homeworkmarket.com/sites/default/files/qx/14/12/16/10/manufacturing_proces s_analysis_with_support_of_workflow_modelling_and_simulation.pdf.

[17] Carlos Monsalve. Business process modeling with levels of abstraction. IEEE COLCOM 2015, (9781-4799-8834-1), 2015. URL http://publicationslist.org/data/a.april/ref-488/PID3675307.pdf. 
International Journal of Software Engineering \& Applications (IJSEA), Vol.8, No.5, September 2017

[18] John G Mooney, Vijay Gurbaxani, and Kenneth L Kraemer. A process oriented framework for assessing the business value of information technology. ACM SIGMIS Database, 27(2):68-81, 1996. URL http://escholarship.org/uc/item/7b04v3t6.

[19] HansWNissen and Georg V Zemanek. Knowledge representation concepts supporting business process analysis. In Working Notes of the KIâ95 Workshop: KRDB-95 Reasoning about Structured Objects: Knowledge Representation Meets Databases, page 32. Citeseer,1995. URL http://citeseerx.ist.psu.edu/viewdoc/download?doi=10.1.1.28.2442\&rep=rep1\&type=pdf\#page=44.

[20] Enrique Silva and Yves Chaix. Business and it governance alignment simulation essay on a business process and it service model. In Hawaii International Conference on System Sciences, Proceedings of the 41st Annual, page 434. IEEE, 2008 URL https://pdfs.semanticscholar.org/6f31/8fbd27797cbe64935491dbf8d72059973b61.pdf.

[21] Patrik Spieß, Dinh Khoa Nguyen, Ingo Weber, Ivan Markovic, and Michael Beigl.Modelling, simulation, and performance analysis of business processes involving ubiquitous systems. In Advanced Information Systems Engineering, pages 579-582. Springer,2008. URL https://www.ibr.cs.tu-bs.de/dus/publications/Caise08.pdf.

[22] Wenan Tan, Fujun Yang, Song Li, Wei Xu, and Yi Du. A Framework for Business Process Simulation Based on Multi-Agent Cooperation. INTECH Open Access Publisher, Vienna, Austria, February 2009. URL http://cdn.intechopen.com/pdfs-wm/6115.pdf.Multiagent Systems.

[23] Wil MP Van Der Aalst, Arthur HM Ter Hofstede, and Mathias Weske. Business process management: A survey. In Wil M.P. van der Aalst, Arthur ter Hofstede, Mathias Weske, Gerhard Goos, Juris Hartmanis, and Jan van Leeuwen, editors, Business process management, Lecture Notes in Computer Science, pages 1-12. Interational Conference BPM, Springer, Einhoven, The Netherlands, 2003.

[24] Wil MP Van der Aalst and Boudewijn F van Dongen. Discovering workflow performance models from timed logs. In Engineering and Deployment of Cooperative Information Systems, pages 45-63. Springer, 2002. URL http://tmpmining.win.tue.nl/_media/publications/aalst2002b.pdf.

[25] Kostas Vergidis, Ashutosh Tiwari, and Basim Majeed. Business process analysis and optimization: Beyond reengineering. Systems, Man, and Cybernetics, Part C: Applications and Reviews, IEEE Transactions on, 38(1):69-82,2008. URL http://ceit.aut.ac.ir/ sa_hashemi/My\%20Teachings/MSCEIT-Business\%20Process\%20Re-Engineering/Resources/101109TSMCC2007905812.pdf.

[26] Jan Vom Brocke, Jan Recker, and Jan Mendling. Value-oriented process modeling:integrating financial perspectives into business process re-design. Business Process Management Journal, 16(2):333-356, 2010. URL http://eprints.qut.edu.au/26230/1/c26230.pdf.

[27] Currie Weerakkody. Integrating business process reengineering with information systems development; issues \& implications. In Wil M.P. van der Aalst, Arthur ter Hofstede, Mathias Weske, Gerhard Goos, Juris Hartmanis, and Jan van Leeuwen, editors, Business Process Managemment, Lecture Notes in Computer Science. Interational Conference BPM, Springer, Einhoven, The Netherlands, 2003.

URL http://www.bus.iastate.edu/nilakant/MIS538/Readings/Integrating\%20BPR\%20with\%20ISD.pdf.

[28] Sören Witt, Sven Feja, Andreas Speck, and Christian Prietz. Integrated privacy modeling and validation for business process models. In Proceedings of the 2012 Joint EDBT/ICDT Workshops, number 78-1-4503-1143-4/12/03, pages 196-205. ACM,Berlin, Germany, March 2012. URL http://www.mathcs.emory.edu/ lxiong/pais12/pdf/PAIS12_Paper6.pdf. 
International Journal of Software Engineering \& Applications (IJSEA), Vol.8, No.5, September 2017

[29] Thomas Zink. Class for aircc journal submissions, 2012. URL https://github.com/72Zn/Templates/blob/master/packages/aircc.cls. This is an unofficial Latex class for Authors of AIRCC Papers. Access timestamp: 2016-10-19. 